\title{
Forthcoming in Synthese
}

\section{Dynamical versus structural explanations in scientific revolutions ${ }^{1}$}

\author{
Mauro Dorato, Department of Philosophy, Communication and Media Study \\ Via Ostiense 234, 00144, Rome, Italy \\ dorato@uniroma3.it
}

\begin{abstract}
By briefly reviewing three well-known scientific revolutions in fundamental physics (the discovery of inertia, of special relativity and of general relativity), I claim that problems that were supposed to be crying for a dynamical explanation in the old paradigm ended up receiving a structural explanation in the new one. This claim is meant to give more substance to Kuhn's view that revolutions are accompanied by a shift in what needs to be explained, while suggesting at the same time the existence of a pattern that is common to all of the discussed case-studies. It remains to be seen whether also quantum mechanics, in particular entanglement, conforms to this pattern.
\end{abstract}

Key words: Scientific revolution, causal explanation, dynamical explanation, structural explanation, Kuhn, inertia, special relativity, general relativity, spatiotemporal symmetries, entanglement

\section{$\S 1$ Introduction}

As is well known, in Kuhn's view scientific revolutions are accompanied by radical shifts in the kind of phenomena that are regarded as in need of an explanation. Unfortunately, he did not specify the nature of such explanatory shifts, but gave only some examples (Kuhn 1970, p. 104, and Kuhn 1977, p. 29). In this paper I want to fill this lacuna by claiming that the three scientific revolutions in fundamental physics that I will discuss share a common pattern that has not been recognized so far, and that differs in some notable respects from Janssen's model of Common Origin Inferences (Janssen 2002a, 2002b). ${ }^{2}$ The essential features of such a pattern correspond to the main thesis of this paper:

\footnotetext{
${ }^{1}$ I thank the two anonymous referees for helpful criticism and suggestions. I also thank the audience in Montreal

${ }^{2}$ In the revolutionary cases that he analyses, Janssen (2002b) is reluctant to identify Common Origin Inferences with Common-Cause Inferences, although in his (2002a) he seems definitely inclined to do so. My view is that causation did not play any role (at least in one sense of "causation") in the discovery of the new theories.
} 
$\mathrm{T}$ : The discovery of inertia, of special relativity and of general relativity has been characterized and made possible by the fact that physical phenomena that were previously regarded as crying out for a (particular type of) causal explanation ended up receiving a structural explanation in terms of suitable components of a new geometrical model of the phenomena.

The plan of the paper is as follows. In the next section $(\S 2)$, I define what I mean by causal explanations and how they relate to dynamical explanations. In (§3) I clarify the notion of structural explanations I will be referring to, and how it relates to causal explanations. A brief discussion of the question whether structural explanations really explain will allow me to shed light on how they could relate to fundamentality in physics. This will require also raising the question whether the common pattern that I identify in the three revolutions supports a particular theory of scientific explanation $(\S 4)$. In the three ensuing sections I illustrate and defend my main thesis by discussing the three above-mentioned revolutionary changes, namely the postulation of inertial motion versus previous causal explanations of Aristotle’s “violent motions” (§5), Einstein's kinematical treatment of the relativistic effects in contrast with previous attempts to derive them from the Lorentz covariance of dynamical laws governing the inner behavior of rods and clocks ( $(6)$, and Einstein's postulation of a curved spacetime versus previous explanations of gravity involving a force $(\S 7)$.

My aim in these three sections is to illustrate the central role of structural explanations in post-revolutionary theories of fundamental physics. Since the historical details of these case studies are well known and - with the exception of those concerning special relativity not particularly controversial, ${ }^{3}$ I can afford to ignore them. It is only the hypothesis that these three case studies exemplify the common pattern specified by $\mathrm{T}$ above that needs to be defended.

Before beginning, it is worth stressing that even though I suggest a general thesis about all of the case studies I discuss, other instances of revolutionary change might not conform to the pattern illustrated here. While I do not claim full generality, the revolutions I discuss are certainly important. It remains to be seen whether $\mathrm{T}$ can be extended to other major transitions

\footnotetext{
${ }^{3}$ The problem is whether relativistic contractions and dilations, and other experimental phenomena concerning relativity should be understood dynamically, as Brown (2005) and Brown and Pooley (2006) have it, or kinematically, as Balashov and Janssen (2003), Norton (2008), Janssen (2009) and Dorato and Felline (2010) have it. Here I will simply assume that the kinematical reading is correct.
} 
in the history of physics, ${ }^{4}$ in particular to quantum mechanics and to explanations of quantum entanglement.

\section{$\S 2$ Causal and Dynamical Explanations in Physics}

The notion of causal explanation that I will be referring to below is as "metaphysically" deflationary as possible. ${ }^{5}$ One way to achieve this minimalistic aim is to claim that in physical theories some causal explanations are given in terms of a dynamical account of the relevant phenomena. A dynamical account involves forces.

It is only to this kind of causal explanations that my thesis T refers, but this restriction is quite plausible. For instance, in classical mechanics the cause that explains the falling of objects is the force referred to by Newton's gravitational law, which is the main dynamical law of the theory. In fluid dynamics, it is the resistance of the air that causes parachutes to fall at constant speed. The cause in question is a force mentioned in the relevant law that is directly proportional to the velocity with which the object moves in the fluid. Analogously, the causal relation existing between like or unlike charges is given by Coulomb's force, while the cause that explains the deflection of charges moving in a magnetic field is the force occurring in Lorentz's equation.

On the basis of these well-known examples, it seems reasonable to assume that to the extent that in physics there are causal explanations, at least some of them make reference to physical forces. The reason for this claim might be that all physical forces are kind of causes, or involve causal relations (Bigelow, Ellis, Pargetter 1988, p. 623). Fortunately, my thesis about the common pattern exemplified by the three case studies below does not depend on the much more controversial claim that all causal explanations in physics are dynamical explanations.

However, there are some reasons, that I don't need to defend, that might suggest trying to reduce - at least in physics - causal explanations to dynamical explanations yielded by the relevant laws. The concept of force is part and parcel of the language in which physical theories and physical laws are expressed - despite eliminationist attempts by Hertz (1894) and it is understood quite well. It is much more controversial to advance similar claims for the concept of cause, not only for the reasons that have been advanced already by Russell (1912),

\footnotetext{
${ }^{4}$ The extendibility to other natural sciences seems an even more remote possibility.
} 
but in view of the current lack of agreement about what causation is from a metaphysical viewpoint. $^{6}$

The reason why I do not need to deny that in physics there might be kinds of causal explanations that do not appeal to dynamical laws can be stated simply: what matters for my purpose is that in the historical episodes that I want to illustrate, the notion of "cause" that was in play was equated with that of "force". Of course, the revolutionary changes that I am about to illustrate could be subsumed under different theories to causation, and I will comment on the most natural such reconceptualizations, independently of the historical settings that I will be referring to.

\section{$\S 3$ Structural explanations}

A first motivation for the recent interests in the role of mathematical explanations of physical phenomena has come from the philosophy of quantum mechanics. If embedding quantum phenomena in appropriate mathematical models provided some form of understanding via such models, we could somehow - at least temporarily - alleviate the remarkable difficulties in providing an agreed-upon ontological picture of quantum mechanics (Hughes 1989a and 1989b, Clifton 1998 and Dorato and Felline 2011). Of course, a generic embedding of physical phenomena in a mathematical model may not suffice to provide a purely mathematical explanation. According to Hughes (1997), for example, the candidate explanans in the mathematical model should also be re-interpreted in physical terms, and therefore, possibly, in a causal language.

A more promising way to defend the role of mathematics in explaining physical phenomena comes from topology, and therefore from non-metrical relations between points that in part involve our spatial intuition: Königsberg bridges (Pincock 2007) and knots (Kitcher 1989 p. 426 and Lange 2013b, p. 490) are an example. In case like these, the geometrical representation is immune from particular causal interpretation, since it applies to any possible instantiation by physical objects of the abstract topological relations. It is this kind of geometrical explanations that comes closer to what I am after: as we will see, what I

\footnotetext{
${ }^{5}$ For a thorough and extremely clear introduction to metaphysical theories of causation, see Schaffer (2009).

${ }^{6}$ As Norton has it: "If we demand a causal explanation that draws on a primitive notion of causation, then we are conjuring up a dubious level of causal metaphysics that must be supposed to be antecedent to science. Successful explication of that notion of cause has eluded us for millennia" (2008, p. 824, n.5). For a contrary view, see Frisch (2009).
} 
label "structural explanation" involves purely geometrical notions like, for instance, affine structure, projections, sections, curvature, etc.

This preliminary account, however, needs to be further refined, also in order to contrast it with the typical kind of dynamical explanations of phenomena that, according to my thesis, have been abandoned by the post-paradigmatic theories to be discussed below. One way to characterize such a contrast would be to define structural explanations in a purely negative manner. However, if a structural explanation were defined just as an explanation that does not appeal to forces, my claim would not be particularly illuminating: it would correspond to claiming that whenever a dynamical explanation was effectively abandoned, the replacement by an (allegedly genuine) structural explanation was equivalent to the abandonment of a dynamical one. A stronger and more general negative characterization should then be obtained by contrasting structural explanations with any possible kind of causal explanations of physical phenomena (despite disagreements about what causation is). In the literature, this position is widely shared (see, among others, Mancosu 2008).

Nevertheless, it might be objected that the claim that a structural explanation is one that does not locate the events to be explained in the causal structure of the world is a purely necessary condition. To the extent that a more positive characterization is needed, a sufficient condition for having a structural explanation should rather make reference to geometrical considerations that appeal to the symmetries defining the geometry of a given spacetime. This approach is much more suitable for discussing the revolutions to be presented below, since they involve a drastic change in our understanding of space and time. In view of the fact that all the physical theories that I discuss presuppose a certain spatiotemporal structure, $a$ structural explanation can be defined as an explanation mentioning the symmetries of the spacetime in which the post-paradigmatic theory is expressed. ${ }^{7}$ It in this sense that such explanations are essentially geometrical in nature.

Furthermore, the term geometrical, at least in spacetime theories, can be further clarified. As noted by Janssen, a distinction between the geometrical/kinematical language of the theory and its physical/dynamical language is very clear, to the extent that there is a sharp separation between absolute geometrical objects - where absolute "means independent of physical objects") and physical entities (Janssen 2009, p.27). ${ }^{8}$ This independence is crucial if a structural explanation in terms of absolute geometrical concepts can be given, since it entails

\footnotetext{
${ }^{7}$ Here the choice of terminology can be misleading: McMullin (1978, p. 139) defines structural explanations by referring to causal language.

${ }^{8}$ The distinction is widely used in the philosophy of space and time at least since Friedman (1983).
} 
the existence of geometrical constraints (say, invariance under automorphisms) that any possible dynamical or causal law must satisfy.

The independence in question holds in particular for the first two case studies that I discuss, which presuppose a fixed spacetime (Newtonian spacetime and Minkowski spacetime respectively). But even general relativity, in which spacetime can (although controversially) be identified with a variable metric field, ${ }^{9}$ does not prevent explanations of the gravitational phenomena that are given in terms of the symmetries of a general relativistic spacetime. The question whether the constraints given by spacetime symmetries can also be interpreted dynamically must be tackled in the particular case studies below, although my claim should already be convincing even from this preliminary characterization. The relativity principle does not hold because of particular dynamical or causal laws; on the contrary, the particular laws have the character they have in virtue of a common geometrical constraint that they must satisfy.

$\S 4$ The genuinity of mathematical explanations and physical fundamentality

Do mathematical explanations really explain physical phenomena? Bueno and French (2012) argue that they don't, Batterman (2010) and (2013b), among others, endorse them, though for different reasons. After having clarified what I mean by structural explanations, luckily I don't have to solve this problem, since my thesis is valid in both alternatives.

Suppose structural explanations are not genuine, so that explanations of physical phenomena can only be given in terms of physical laws or causal processes. In this hypothesis, as we will see in more details below, the post-paradigmatic structural explanations "explain" by showing that there is nothing to be explained! Such a dissolution of the whyquestion amounts to regarding the phenomenon as physically fundamental, or "natural" in the sense of Aristotle. From this perspective, the thesis that scientific revolutions entail an explanatory switch from a dynamical to a geometrical explanation can be clarified as follows. In the post-revolutionary theory, a geometrical "explanation" (i.e. its redescription in mathematical language) of a phenomenon $P$ amounts to regarding $P$ as "natural", ${ }^{10}$ while a new dynamical explanation is called into play only when there is a deviation from the newly discovered natural order.

\footnotetext{
${ }^{9}$ According to various philosophers, a general relativistic spacetime is to be identified with the manifold, since the metric field is a "physical" field (Belot and Earman 2001).

${ }^{10}$ For this sense of "natural", see Achinstein (1983, p. 291) and Toulmin (1961, p. 45).
} 
In this sense, in Aristotelian physics vertical motions were natural, violent motions were not. With Newton, inertial motion became the new natural motion of bodies, and it is only a deviation from this motion that required a dynamical explanation. Before Einstein's theory of special relativity, contractions of rods and dilations of clocks were regarded as in need of a dynamical account, after the revolution they became natural, since these frame-relative effects can be redescribed geometrically in terms of sections and projections within Minkowski's four-dimensional spacetime (Dorato and Felline 2010). Finally, in general relativity free fall becomes natural and does not require dynamical accounts, and it is only deviations from free fall that need to be explained in terms of the presence of masses. ${ }^{11}$

If, alternatively, we granted structural explanations a genuine character, we would have two possible views, a weaker and a stronger one. According to the former, a geometrical/structural explanation does offer some form of "understanding" by providing a different conceptual perspective on the explanandum. At the same time, however, such an understanding does not provide a deeper physical explanation, for the simple reason that in the context of the new theory it cannot be found. This weaker view, therefore, is not so different from the one considered before about the naturalness of, say, the Lorentz contractions and dilations, and has been advocated by Di Salle: "We can certainly think of this [geometric] representation as an explanation of a sort, provided that we don't confuse understanding a theory from a different viewpoint with deriving it from some deeper ontological ground" (Di Salle 2006, p.116). The main problem with this weaker view of structural explanation is, of course the vague, and psychological notion of "understanding" (see Achinstein 1983, and De Regt, Dieks 2005 for an attempt to clarify it).

A stronger explanatory viewpoint has been defended by Lange (2009, 2013a, 2013b), and can be cashed out in terms of the modal character provided to causal laws by geometrical explanations in terms of the symmetries of spacetime. According to Lange, distinctively mathematical explanations explain "by (roughly) showing how the fact to be explained was inevitable to a stronger degree than could result from the causal powers bestowed by the possession of various properties" (2013b, p. 487-491). If this were the case, structural explanations as characterized in the previous section would explain by conferring to the physical phenomena a sort of necessity that they don't possess; the necessity in question

\footnotetext{
${ }^{11}$ Interestingly, also Brown, despite his position on dynamical accounts of relativistic effect, agrees with me in thinking that the distinction between natural and non-natural motions has an important role to play in the history of physics: "GR is the first in the long line of dynamical theories, based on that profound Aristotelian distinction between natural and forced motion, that explains inertial motion" (2005, p.141).
} 
depends on the fact that the spacetime symmetries, among them the relativity principle in mechanics and electromagnetism and the equivalence principle in GTR, are "meta-laws" (Lange 2009).

However, despite the fact that (i) I don't need to choose between the weaker and a stronger sense presented above, and that (ii) I agree with Lange on the importance of symmetries in structural explanations, ${ }^{12}$ it is important to note that a stronger view about the explanatory power of the latter does not necessarily involve modal claims about degrees of necessity. On the contrary, the case studies presented in the next three sections seem to be better supported by an unificationist account of explanation (see below).

In a word, when the structural "explanation" dissolves the need for explaining, or provides nonetheless some understanding in terms of a reformulation of the previous causal problems in mathematical terms (the weak view), the physical phenomena that are "explained" by the post-revolutionary theory become natural, that is, fundamental. If explanations provided in terms of spacetime symmetries explained instead in the strong sense, the explanans would count as fundamental in virtue of the fact that it is a "metalaw", i.e., a geometric constraint on all physical or causal laws. In both alternatives, what is important for my purpose is that in the scientific revolutions that I am about to sketch, structural explanations can be seen either as geometrical redescriptions of fundamental physical phenomena or as themselves fundamental.

To conclude this section, let me stress that the theory of explanation that is presupposed (as well as illustrated) by the historical examples below is not committed to a particular view of scientific explanation. However, it is certainly closer to the contextual view (De Regt and Dieks (2005) and, as anticipated above, to the unificationist view (Friedman 1974 and Kitcher 1989). It is contextual because the fact that some phenomena are regarded as crying out for an explanation and some aren't depends holistically on the web of beliefs (Quine and Ullian 1978) of the scientific community asking the why-questions, and this web obviously changes with the historical context. It is unificationist because, as we will see, if structural explanations provide understanding, then they do so by unearthing a common structural pattern exemplified by all the relevant physical explananda.

$\S 5$ From impetus to inertia: the first case study

\footnotetext{
${ }^{12}$ For the importance of structuralist understandings of spacetime physics, see also North $(2009$, p. 68$)$
} 
Since according to Aristotle each violent motion requires a mover that be in causal contact with the moved object, one of the greatest problems of Aristotelian and postAristotelian physics till Galileo and Descartes was that of explaining the continuation of motion in the absence of a visible contact between the original mover and the moved object. If we don't presuppose inertia, the fact that an arrow keeps moving after it has left the bow needs an explanation. In the Aristotelian tradition, there were two rival causal accounts: either the air pushed by the tip of the arrow moves circularly behind it, thereby pushing the tail, or the air receives from the original movers (the hand and the bow) the power to act as a mover, so as to move the arrow also after it has left the bow (see Clagett 1961). Even later impetus theories (Buridan) postulated a hidden causal power that would continue to act forever on the arrow, and in the initial direction of motion, if friction and weight would not alter its action (see Grant 1977, Thijssen and J. Zupko 2001).

This extremely sketchy historical overview is nevertheless sufficient to illustrate my thesis: the revolutionary transition to modern dynamics was achieved by transforming a particular kind of causal problem (i.e. a dynamical problem) into a structural explanation, provided in this case by a new fundamental law of nature of geometrical character. ${ }^{13}$ Whatever in the pre-modern physical theories constituted a problem - for their continuation violent motions required explanations in terms of some sort of hidden causal power- with Galileo, Descartes and Newton becomes part of a new "natural" order in the sense specified above. In the absence of forces, bodies travel with the same speed in the same direction "naturally", where naturally means, as specified above: "no further physical explanation given in terms of forces is to be expected". What needs to be explained after the postulation of inertia is rather why bodies do not continue moving in a straight line with constant speed, and for that we need a causal notion, a force, which brings about either a change of direction to the vector representing velocity, or simply a change in speed. The new classification between what needs a causal explanation and what doesn't constitutes the core of this and other scientific revolutions to be discussed later, a fact described by Janssen in terms of the distinction between kinematical and dynamical account of phenomena (2002a, 2009).

I argue that it is important to add to his account that those phenomena that after the revolution are postulated as being physically primitive can be understood or explained - recall the above distinction between weaker and stronger views of structural explanations - via a

\footnotetext{
${ }^{13}$ If laws are postulates, a quotation from Goethe taken from a letter written on 9.8.1828 to his friend Karl F. Zelter becomes relevant: «the greatest art in the life of the world and of culture consists in the ability to transform a problem into a postulate.»
} 
geometrical model. In particular, it is the association between inertial and rectilinear motion that explains inertia structurally and geometrically. The structural element in the explanation of inertia is revealed by the fact that in the absence of forces, and given that velocity is mathematically represented by a vector, the direction and intensity of the vector representing an inertial system is conserved: it is only the change in the intensity or direction that needs a causal account in terms of forces.

The structural explanation of inertia is realized via a unification: the geometrical structure common to all inertially moving bodies is defined in terms of the global symmetries of Galilean spacetime and its invariance under rotations, translations and boosts. The unification afforded by the geometrical explanation therefore depends on two related factors; the first is the universality of the spatiotemporal symmetries, exemplified everywhere and at all times by all inertial systems. The second explains why such symmetries apply to all inertially moving bodies: the former are obviously independent of the concrete physical or chemical composition constituting the latter. In a word, the structural explanation of inertia unifies all the relevant physical explananda by unearthing the common structural pattern that they exemplify.

In more sophisticated and even more anachronistic language, inertial motions can be understood in terms of their representative in the appropriate mathematical model, namely the affine structure of a neo-Newtonian spacetime (Earman and Friedman 1973), whose geodesic are the trajectories of inertially moving particles. ${ }^{14}$ It is the reference to such geometrical descriptions that makes the explanation structural and not causal, in any possible sense of causal (not just in the dynamical one). In particular, as pointed out by Lange, the different lower-level causal laws obeyed by physical bodies with their different constitution do not need to be mentioned in what I take to be the unifying language in which geometrical explanations are framed.

A point here is worth noting, in particular against a common misinterpretation of structural explanations in the case of spacetime theories. It does not make sense to claim that the structure of neo-Newtonian spacetime causes force-free particles to follow straight lines (see also Nerlich 1994, 2010). In full agreement with Brown and Pooley (2006), neoNewtonian spacetime "is not a substance with causal efficacy", as Janssen efficaciously put it a propos of Minkowski spacetime $(2009$, p. 28). Since (i) the discovery of inertia entailed the replacement of dynamical explanations with a structural account, and (ii) some causal 
explanations are dynamical, a structural understanding or explanation of inertia is, to some extent, non-causal. ${ }^{15}$ In agreement with Brown, force-free particles do not follow "grooves" of spacetime along which the latter "nudges" them (Brown 2005, p.24).

In a word, with the demise of Aristotelian physics the natural order of things (what needs to be explained) gets transformed, even though the Aristotelian distinction between natural and non-natural motions keeps an essential role also in modern physics. As a matter of fact, it becomes the distinction between rectilinear (natural) and accelerated (non-natural) motion, a distinction that in the Newtonian context must be regarded as objective.

Therefore, already in this case we see an exemplification of my main thesis: revolutionary changes in physical theories can be described as a change in the class of phenomena that are regarded as natural or physically primitive. In Aristotelian physics, free fall was a natural motion and it was the continuation of motion (violent motions) that needed special causes. With Newton, the continuation of motion becomes natural and it is a deviation from it that requires causes (the gravitational force) ${ }^{16}$ From what was said above, it should be clear why the naturalness or fundamentality of inertia from the physical point of view does not mean that it cannot be understood or explained geometrically via the affine structure of spacetime. If structural explanations explain, they do so in terms of this structure, which unifies the behaviors of inertially moving bodies by showing the common feature that they all instantiate.

There are at least three objections to a merely structural explanation of inertial motion that need to be discussed, since they are going to be relevant also for the next two case studies.

O1) In order to defend my thesis, in section 2 I stressed the importance of the historical language in which the new theories have been formulated. However, according to Newton (and to some contemporary readers), bodies are endowed with a vis inertiae, i.e., with both a resistance to being accelerated - which is what we today call "inertial mass" -, and with an impetus that endeavors to change the state of an obstacle that the body may encounter (Newton 1726 [1999], pp. 404-405). According to this objection, even according to Newton

\footnotetext{
${ }^{14}$ Roughly, an affine space is a vector space from which one removes the origin. A vector is therefore specified up to translations.

${ }^{15}$ Possible generalizations of this claim will be discussed in the remainder of this section.

${ }^{16}$ The question that I cannot discuss in this context is whether the gravitational law ought to be regarded as structural or causal. According to Kuhn, Newton's law of gravitation was initially received as a merely structural explanation, especially among the followers of Descartes, but physicists later got used to it: "[. . .] in physics new canons of explanation are born with new theories on which they are, to a considerable extent, parasitic". (Kuhn 1977, p. 29).
} 
all bodies have well-defined causal powers (resistance and impetus) that explain with a causal language why they "tend" to keep moving in a straight line, whenever they are undisturbed by forces. From this viewpoint, not only would the disposition to move inertially be the truthmaker of the first law, which would otherwise be non-exemplified in the real world; at the same time, this disposition could be identifiable with the scientifically respectable notion of inertial mass (resistance to acceleration), which, contrary to my thesis, seems to have dynamical implications.

O2) On the basis of the Deductive Nomological model, the first law can be explained from the second, of which it is a particular case. Therefore, it might be argued that also the law of inertia, being grounded in a dynamical law, has a dynamical content that refers to the causal structure of the world: after all, a body is at rest or continues to travel with the same velocity because there is no force acting upon it.

O3) The third objection raises the possibility that inertia can be explained both structurally and causally. There are two theories of causation that at this point need to be distinguished, since they have conflicting consequences on the interpretation of the law of inertia. According to the fist theory, an explanation is causal only if it refers to causes acting directly on bodies (see Colyvan 1998, pp. 324-5). Since unaccelerated motion is associated with no net force impressed on a body $B$, in this sense of causation the absence of forces acting on $B$ implies the absence of causes, and therefore the unavailability of a causal and dynamical explanation of $B$. After all, "omissions" (in our case, the absence of forces on B) cannot be part of real causal processes, since omissions are not real events.

The second, counterfactual theory of causation, on the contrary, can cite omissions: as such, according to Brandon (2006) and (2013b), it can refer to the net of real causes and effects. Brandon, for example, argues that when we explain why a given body is moving uniformly by citing the absence of forces, we give a sort of "default causal explanation" (Brandon 2006, p. 321). While the uniform motion of a body feeling no forces has no acting causes in the sense of Colyvan, such a motion has nevertheless a causal explanation, because it refers to the dynamical structure of the world. This is evident if we recall the well-known formulation of the Principle of Inertia: "a body is in an inertial state unless it is acted upon by a force". By referring to what would happen if we intervened on an inertial system by impressing a force upon it, this formulation introduces a reference to forces. As such, this explanation of inertia is dynamical, and is therefore a refutation of my thesis.

Let me briefly reply to these objections in turn. 
R1) First of all, a well-know historical fact is relevant here: the first exact formulation of the principle of inertia was due to Descartes, whose physics explicitly rejected any recourse to hidden forces, ${ }^{17}$ regarded by him as a remnant of a magical and anthropomorphic conception of the world. Recall that here I am interested in the context of discovery and therefore in the way causal explanations were used in the historical period I am referring to. Consequently, it is important to take into account that, according to Descartes, dynamical explanations of inertia could not exist, and that the only change in the motion of bodies was due to impacts.

Furthermore, according to Newton resistance and impetus only intervene when some force is applied (and inertial motion in these cases is absent). It follows that in such cases it is the Principle of Action and Reaction that comes into play (Newton, 1726, ibid.). Even if one treated inertial mass as a disposition (a fundamental property of massive bodies), and even granting that dispositions explain, what is in question in our case is what explains inertial motion, rather than what explains the inertial mass of bodies. And the disposition to move in a straight line with the same speed, unlike what happens in Aristotelian physics, makes no reference to forces acting on the body. I submit that if we missed this point, we would miss the great novelty of the scientific revolution of the modern age.

R2) To the extent that inertial motion is the natural state of physical bodies, the first law is explanatorily more fundamental than the second, since what needs to be explained in Newtonian physics is not why bodies move inertially (a primitive, natural fact), but rather why they typically don't do so due to the intervention of forces.

Furthermore, two things need to be noted about the DN explanation given above. The first is a clarification: the deduction of the first law from the second is mathematical but the simple mathematics involved has nothing to do with the kind of structural explanation advocated above: when we use the second law in its differential form, $\boldsymbol{F} d t=m d \boldsymbol{v}$, the absence of net forces, $\boldsymbol{F}=0$, entails the constancy of $\boldsymbol{v}$. The second comment stresses the fact the explanatory power of the second law with respect to the first springs from the purely mathematical fact that whenever the first derivative of a function is 0 , the function $(v)$ is constant. However, the fact that this explanation of the first law consists in a mere mathematical derivation shows that a DN explanation of the first law from a dynamical law does not suffice to claim that the also the former has a dynamical content. In order to provide further arguments against the view that the law of inertia (more or less implicitly) makes

\footnotetext{
${ }^{17}$ I owe this point to one of the referees.
} 
reference to the dynamical structure of the world, we must move to the reply to the third objection.

R3) In order to rebut O3, I will put forward the following three replies, which will also allow me to circumscribe my claim more precisely.

R3.1 Do counterfactual theories of causation entail dynamical redescriptions of the principle of inertia? If this were the case, the continuity between the Aristotelian worldview and ours would be much more pronounced than my thesis would make us believe. The crucial point in order to answer the above question is to note that a body, as Newton himself wrote in his fourth letter to Bentley, cannot act "upon another at a distance through a vacuum, without the mediation of anything else, by and through which their action and force may be conveyed from one to another" (Janiak 2004, p.102). ${ }^{18}$ With later language, we would say that even according to Newton, the manifestation of forces requires a process theory of causation (Dowe 2000), in which causation is a continuous $^{19}$ process that is capable to describe any interaction among physical systems.

On the contrary, counterfactual theories of causation do not seem suitable to treat dynamical interactions, given that they allow for causes that are spatiotemporally disconnected from their effects (Schaffer 2000, Hall 2004). Consequently, in view of the context of discovery of the principle of inertia, and its possible reconstruction in contemporary language, counterfactual theories of causation do not seem to threaten my main thesis. To recapitulate this reply with Janssen's wording (2009), the transition from preAristotelian physics to Galilei's and Descartes' was a reclassification of the problem of continuation of motion from a dynamical to a kinematical category, where the latter term, in my reading, refers to the symmetries of Galileian spacetime.

R3.2 This is also confirmed by an extremely sketchy historical consideration. In trying to account for the transition from the Aristotelian worldview to the modern one, the notion of causation that was involved is efficient or direct causation, in which the cause acts on the effect only when it is in contact with it. In this context, counterfactual causation played no role. According to Aristotle, in fact, there is an objective distinction between a body at rest and a body travelling at constant velocity. The former requires no efficient causation in the

\footnotetext{
18 "It is inconceivable that inanimate brute matter should, without the mediation of something else which is not material, operate upon and affect other matter without mutual contact... That gravity should be innate, inherent, and essential to matter, so that one body may act upon another at a distance through a vacuum, without the mediation of anything else, by and through which their action and force may be conveyed from one to another, is to me so great an absurdity that I believe no man who has in philosophical matters a competent faculty of thinking can ever fall into it" (Janiak 2004, p. 102)
} 
form of a mover, the latter does. In modern physics, the distinction is completely lost, in virtue of the indistinguishability between rest and uniform rectilinear motion: the new objective distinction is between non-accelerated and accelerated motion. In this restricted sense of causation, inertial motion is explained causally only if there is a force actually acting on the body. This is all that matters for my thesis, despite the fact that inertia might be explained counterfactually by a different theory of causation.

R3.3 Suppose that, by following Woodward's approach, we could advance the following claim: "if the gravitational force linking the Earth to the Sun were removed by an imaginary manipulation, our planet would continue to move forever with the same velocity, so as to exemplify inertial motion". In order for this claim to count as a causal explanation of inertia (with allegedly dynamical consequences), the merely logical possibility of an intervention must suffice. However, this assumption is per se rather problematic (see also Reutlinger 2012 for additional arguments). In view of the fact that the force of gravity cannot be screened off, a follower of Woodward should grant the physical impossibility of manipulations of the kind envisaged above, even if it were a non-anthropomorphic one. For instance, a gigantic black hole swallowing the Sun would change also the Earth's trajectory. However, since the truth of the counterfactual above presupposes that there is a logically possible world in which any dynamical interaction between the Sun and the Earth is cancelled, my thesis is not jeopardized. In that logically possible world $W$ in fact, the Earth's inertial motion inertia cannot be accounted for dynamically, precisely because in $W$ the Sun's gravitational force is removed.

$\S 6$ The Special Theory of Relativity: the second case study

The above-illustrated switch from attempts at explaining puzzling phenomena in terms of dynamical hypotheses to post-revolutionary redescriptions or explanations in terms of spatiotemporal symmetries is even clearer in the case of special relativity. In view of the fact that the light quantum hypothesis, put forward by Einstein in 1905, had already convinced him that Maxwell's equation "could not claim to exact validity", the Light Postulate of special relativity (the fact that light has a velocity that is independent of the motion of the source) had to be formulated independently of any theory about the inner constitution of light (Brown 2005, p.77): such an independence is a first, strong evidence for the presence of structural

\footnotetext{
${ }^{19}$ Of course, this holds for classical, non-quantum forces.
} 
explanations. For exactly the same reason, Einstein had to postulate the Relativity Principle, rather than deriving it from Lorentz covariant dynamical laws involving intermolecular forces. Explanations of these kinds had been attempted by FitzGerald, Larmor, Lorentz and Poincaré, and were dynamical or causal because they depended on attempts to construe a Lorentz covariant theory of the inner constitution of rods and clocks.

In a word, rather than explaining the Light Postulate or the Relativity Principle by postulating some forces, in 1905 Einstein regarded them as physically fundamental. According to my characterization of structural explanations, the transformation of two dynamical problems into two postulates implies that the Light Principle, the contractions of length, the dilations of times, and other experimental evidence mentioned by Janssen (see note 21 below) can only be redescribed or explained in that geometrical language that was later construed by Minkowski.

It seems legitimate to conclude that also in this second case, pre-revolutionary dynamical explanations have been replaced by post-revolutionary structural explanations. A possible objection to this thesis could stress that I am relying on the historically contingent fact that Special Relativity was formulated as a Principle theory and not as a Constructive theory. ${ }^{20}$ The objection could continue by stressing that even though the theory originated as a principle theory, it ought not to be conceived in this way (Brown 2005), so that contractions and dilations should eventually be explained in a causal/dynamical fashion. This seems to have been a possibility envisaged by Einstein himself (1919).

There are three replies to this objection, the first two of which accept the relevance of the principle/constructive distinction for the interpretation of this scientific revolutions, while the last, more plausibly and in accord to Janssen, denies it. My first reply rejects the claim that special relativity ought to be formulated as a constructive theory, for reasons that have been already provided by Balashov and Janssen (2003), Dorato (2007) Norton (2008) and Janssen (2009), and that here will not be rehearsed. ${ }^{21}$ My second reply notes that even supposing that eventually we will come up with some dynamical explanations for rods' contraction and clocks' dilations, the fact that historically things went as my thesis requires for my purpose is sufficient. Any theory of scientific revolutions should be based, in Ranke's famous words, on

\footnotetext{
${ }^{20}$ Recall that the former raises empirical regularities (like those of thermodynamics) to universal principles, while the latter looks for deeper theories (like those of statistical mechanics) that can explain such regularities (Einstein 1919).

${ }^{21}$ Unlike Norton (2008), Janssen (2009) more convincingly does not presuppose realism about Minkowski spacetime, but insists on the fact that experimental results like the Fresnel drag effect, the velocity dependence of
} 
history "as it really went", and not on unpredictable and - in my view - implausible future developments of physics.

For the third reply I can appropriate what has argued by Janssen: "The principleconstructive distinction is a red herring in the end. By focusing on it, Lorentz missed a much more important difference between his own theory and Einstein's, namely the difference between kinematical and dynamical accounts of a whole class of phenomena. It was for a reason that Einstein called the first half of his 1905 paper: "The kinematical part.' (2009, p. 38). ${ }^{22}$ So in any possible case, the objection is rejected.

Here I will assume that Janssen is correct. Since I take it that a first component of the meaning of "kinematical" is equivalent to "independent of specific dynamical assumptions", the historical fact that in 1905 Einstein derived as kinematical effects what Lorentz wanted to explain dynamically is evidence for the first component of my thesis: scientific revolutions are accompanied and made possible by the abandonment of pre-revolutionary searches for dynamical causes. According to Janssen, kinematical also refers to "a generic feature of the world, in [Einstein's] case instances of default spatio-temporal behavior" (Janssen 2009, p. 27). I interpret this second, but more unclear specification of the meaning of kinematical, as evidence also for the second component of my thesis. As I read this quotation, "the generic instances" occurring in it refer to the fact that the physical world exemplifies the geometrical structure of Minkowski spacetime. The behavior, say, of the electromagnetic field, and that of the other physical fields, is described or explained in virtue of the fact that they share a common fourdimensional geometrical structure. And the explanatory force in question derives from a theory of explanation that insists on the view that explaining is unifying different, seemingly and previously unrelated physical phenomena: all mechanical as well as electromagnetic phenomena obey the relativity principle, and therefore exemplify the geometrical structure of Minkowski spacetime. The term "default" in the quotation above also gives evidence to the third aspect of my thesis: scientific revolutions redefine what is physically "natural" (and therefore fundamental) and therefore also what instead needs an explanation in dynamical or causal terms.

In the case of special relativity, the fact that what is physically natural can be explained in structural terms is exemplified by the well-known effects of spatial contraction and time

the electron's mass, and the torques on a moving capacitor in the Trouton-Noble experiment ought to be characterized as kinematical rather than dynamical.

${ }^{22}$ I thank one of the referees for having pointed out to me that the distinction between Principle and Constructive theories is independent of the structural/dynamical one. 
dilation. As is well known, such effects are measurable in hadron colliders, and in this sense they are perfectly real and objective; and yet they are not real in another sense, given by "invariant". It is the fact that the length or the shape of entities (or the temporal intervals between pairs of events) is non-invariant under the full Poincare's group that motivates a structural explanation of the relativistic effects. It is this mathematical fact that renders explanations in terms of constructive, law-covered causes or mechanisms completely unnecessary. Since one can talk about a definite amount of a contraction only relatively to an arbitrarily chosen inertial frame, and since by changing frame we change also the amount of contraction, there is no invariant/objective fact (the contraction) to be explained causally/dynamically. Consequently, spatial contractions are to be understood as crosssections or projections of four-dimensional rods onto different, arbitrarily chosen inertial frames of Minkowski spacetime (Balashov and Janssen 2003, Dorato and Felline 2010, Hughes 1989a), in the same sense in which the affine structure of spacetime gives a geometric account of inertial phenomena. Fully analogous considerations hold for time dilations.

For this reason, Brown's complain that "It is wholly unclear how this geometrical explanation is supposed to work" (2005, p. 134) is unmotivated. However, I agree with him when he claims that Minkowski spacetime is "a glorious non-entity" (Brown and Pooley 2006). In a sense in fact, it is not a substance causing or forcing the spatiotemporal behavior of bodies; in another sense, however, the structure of such a spacetime is "really" exemplified by the entities inhabiting the spacetime (Stein 1989). "Exemplified" has a precise meaning: Brown is correct in arguing that the fact that different substances making up the rigid support of the interferometer all obey the FitzGerald-Lorentz deformation effect needs an explanation (Brown 2005, p. 30). But precisely the independence of the contraction from the particular structure of matter making up the interferometer is evidence that the sought-after explanation is to be found in the constraints that the relativity principle poses to the laws of nature, and therefore in the "unifying" symmetries of Minkowski space-time. ${ }^{24}$

In sum, analogously to the question we raised before a propos of uniform motion, what before the revolution seemed to be in need of a dynamical or causal explanation was accounted for afterwards in structural/geometrical terms!

\footnotetext{
${ }^{23}$ A "tertium-quid" view between substantivalism and relationism points to a sort of structural spacetime realism (Dorato 2000).

${ }^{24}$ Janssen refers to the unification afforded by Minkowski as an explanation that explains all the relativistic effects "in one fell swoop" (2009, p. 49). For similar considerations on the constraints posed to causal laws by metalaws, see also Lange (2013a).
} 
$\S 7$ Einstein’s Principle of Equivalence in general relativity: the third case study

In virtue of Einstein's Principle of Equivalence in its strong form, a test particle in free fall in a homogenous gravitational field is in a state of motion that needs no dynamical explanation. Since in virtue of this Principle free fall becomes locally indistinguishable from inertial motion, it also becomes a very strong candidate to be physically fundamental or natural. It is instructive to note that in Newton's theory inertial motion was natural and therefore explainable only structurally. With the general theory of relativity (GR) instead, inertia becomes explainable via the vanishing of the covariant divergence of the stress-energy tensor (see also Brown 2005, p. 141). This confirms the contextual theory of explanation defended at the end of $\S 4$ : what counts for natural or physically fundamental in a given context can be explained by a successive physical theory in physical, non-purely structural terms.

However, it should be noted that the deductive-nomological explanation of inertial motion in GR crucially does not entail that in this theory the free fall of a body receives a dynamical account in terms of forces. The trajectories of freely falling bodies in GR are no longer regarded - as they were in Newtonian mechanics (leaving aside neo-Newtonian spacetime) - as deviations from states of inertial motion caused by the gravitation force.

In a word, after GR what was previously regarded as being in need of an explanation via the introduction of a gravitational force, namely free fall, becomes fundamental or natural. Also in this third scientific revolution, the physical fundamentality of free fall is equivalent to the fact or at least entails that it is structurally explainable in terms of the new geometry of spacetime, in the same sense in which inertial motion and the relativistic effects of the special theory of relativity were explained structurally by the geometry of Newton and Minkowski spacetime respectively.

More in detail, in the case of GR the geometrical explanation is based on the wellknown fact that a general relativistic spacetime is locally Euclidean for homogenous gravitational field and curved for non-homogenous fields. In the new mathematical model of GR, the concrete paths of bodies in free fall are represented by the geodesics of a new curved connection of spacetime: geodesics are "the straightest possible lines" of a curved manifold. The correspondence between free fall and inertiality, however, is valid only infinitesimally: since geodesics have a relative acceleration and deviate, in virtue of the so-called "geodesics 
deviation equation" it follows that the spacetime to which they belong is curved. ${ }^{25}$ To the extent that in general relativity the geometrical/structural notion of curvature of spacetime replaces the concept of force as an explanation of gravitational phenomena, my main thesis is confirmed also for GR. As in the previous cases, the revolutionary explanatory switch entails that what appeared as puzzling (the deviation from inertial motion realized by free fall) and requiring a force regarded as a particular type causal relation, is now regarded as physically fundamental and therefore explainable only structurally, in the progressively stronger senses of explanation described in $\S 4$.

There are at least three objections to the claims just stated in the previous paragraph.

O1) The first is raised by Salmon's question whether, at takeoff, a helium-filled balloon moves toward the rear or toward the front of the airplane containing it (Salmon 1989, p. 184). However, Salmon claimed that one could explain the phenomenon of the balloon moving forward by using two different accounts, one structural, relying on the Principle of Equivalence, and one citing causal models involving the pressure of the air inside an airplane that moves forward. The existence of a structural explanation, in other words, does not exclude a dynamical explanation in terms of a pressure gradient exerting a force on the balloon.

R1) Despite the correctness of Salmon's example, which refers to the fact that phenomena observed inside a cabin at rest in the Earth's gravitational field are indistinguishable from those observed in a cabin which is accelerated in the opposite direction with $-g$, it remains true that in GR the gravitational force is simply non-existent, so that no dynamical explanation of gravity can be forthcoming.

O2) The second objection exploits the counterfactual account of causation referred to above, and applies it to the current case study.

R2) To the extent that only some causal explanations in physics are given in terms of forces, and my thesis concerns only the replacement of dynamical explanations with structural explanation, I could afford to ignore counterfactual approaches to causation altogether. In any case, even if one insisted in wanting to defend a causal account of free fall in GR in terms of counterfactuals, one should consider the existence of "empty" solutions of Einstein's field equations. As a consequence, for instance, Woodward's counterfactual explanation of gravity could not amount to the claim: "if matter had not been present, spacetime would not have been curved". Curvature can be present independently of the existence of matter and is not a

\footnotetext{
${ }^{25}$ The geodesics deviation equation links the existence of a reciprocal acceleration between geodesics to a non-
} 
causal explanation of gravity, otherwise we would have to accept the already rejected view that the geometry of spacetime "nudges" test particle to follow geodesics.

O3) The third objection is based on the highly plausible remark that in GR the geometrical notion of metric field is identified with the physical notion of the gravitational field. It could therefore be objected that in GR one can hardly distinguish structural from causal explanations. If the explanation provided by the curvature of spacetime is both causal and structural (i.e., given in terms of the geometrical model of a curved Riemannian manifold with Lorentz signature), GR would falsify my claim.

However, there are at least three replies to $\mathrm{O} 3$, which amount to a definite rejection of the objection.

R3.1 The explanatory role of gravitational phenomena in GR is played by a geometrical notion (curvature), defined in terms of the deviation of geodesics, which in its turn refers to another geometric notion representing (in the mathematical model) the trajectory of nondynamical, force-free particles. Importantly, as in two case studies, these geometrical notions are independent of any possible instantiation by concrete physical systems obeying the relevant causal laws. And due to this unification, they explain.

R3.2 The idea of giving a causal explanation of the features of the metric tensor in terms of the matter-energy tensor is fraught with problems. The former tensor is in fact necessary also to define the latter, given that it is present on both sides of Einstein's field equations. The equality sign in these equations therefore should be regarded as a correlation of the two fields and not as referring to causal relations existing between them. The above-mentioned fact that we can have a gravitational field in a curved spacetime without a matter field suggests that matter does not necessarily acts on spacetime by "telling it how to curve".

R3.3 Finally, if the deviation of geodesics is part of the meaning of curvature in virtue of a well-known theorem, why supposing that matter causes spacetime curvature ${ }^{26}$

In a word, the common pattern illustrated in the previous case studies is present also in the revolutionary change leading to GR: answers to why-questions that were given in dynamical terms by the prerevolutionary theory have been replaced by structural explanations based on the symmetries of the relevant spacetime. In the case of GR, such symmetries are given by active diffeomorphism invariance, given that general covariance might have no physical meaning. 
$\S 8$ A brief comparison with Kuhn's view and two open questions

In Kuhn's view, scientific revolutions are essentially characterized by radical shifts in the meaning of key theoretical concepts, which have repercussions in what counts for an adequate explanation of a phenomenon. ${ }^{27}$ On the contrary, here I have argued that the three fundamental scientific revolutions that I have discussed share a common pattern and are the consequence of the same explanatory "switch": in principle, there is no need to assume shifts of meaning. Phenomena that before the revolution seem in need of dynamical explanations, after the revolution become physically fundamental or "natural", and are therefore understood or explained in purely geometrical terms. To the extent that forces belong to the language of physics and are particular types of causal explanations, these scientific revolutions have been accompanied also by the abandonment of causal explanations.

The fact that the scientific revolutions that I discussed above can be characterized by the successful identification of a new natural order of things also explains why philosophy and conceptual analysis have played an important historical role in all of them, in particular to dissolve the explanatory conflict between the new and the old theory. In this respect, the importance of a philosophical analysis of scientific revolutions is also suggested by the following, still unexplored hypothesis. The idea is that some explanations that prevailed in the pre-revolutionary theories might have been suggested by innate views of the physical world. For instance, is the Aristotelian belief that any motion presupposes a mover part of the naïve physics of our brain? Why do we naturally believe that the present moment is cosmically extended, even after the discovery that simultaneity is relative? And more generally, is our belief that there exist forces only grounded in the culturally acquired physics? If these beliefs had been somewhat hardwired in our brains by evolution, a reconciliation of the prerevolutionary and the post-revolutionary explanatory demands would entail that in the three case studies the search for forces has been suggested by our "manifest image of the world" (Sellars 1962). Consequently, the revolutionary steps forward in our knowledge of the world would have been made possible by the realization that a central component of the manifest image was illusory. Such a realization, therefore, could not be interpreted as giving rise to Kuhn's well-known "losses” (Kuhn 1962, pp. 99-100).

\footnotetext{
${ }^{26}$ See also Nerlich (2010, p. 190).

${ }^{27}$ For instance, as a consequence of a radical theoretical change, he noted how, during the $17^{\text {th }}$ century, the search for explanations in terms of occult qualities was replaced by mechanical explanations (Kuhn 1970, p. 104).
} 
The second open question is that the important role played by structural explanations may depend on the fact that all of the three revolutions involved new spacetime theories. A task that is still ahead is to test the pattern suggested here also in the quantum revolution. For instance, we could ask whether attempts to explain quantum entanglement causally might not be completely wrongheaded. What if the quantum correlations between the measurement outcomes of entangled particles don't need any causal explanation (as argued already by Fine 1989)? This hypothesis would fit amazingly well with the pattern of scientific change that I have illustrated so far. Significantly, in this case "causal" would not refer just to explanation in terms of forces, but - due to the randomness of quantum theory - would also extends to counterfactual and "interventionist" accounts of causation. It would then follow that in the quantum case, the explanatory switch illustrated above would entail that rather than trying to explain the quantum non-local correlations in terms of the causal ontology of the previous, classical theory of the world of our experience, we ought to accept them as paradigmatic examples of a new fundamental order of nature. We should therefore presuppose such new natural order in order to explain what we have been taking for granted so far, namely the local, separable nature of the classical world of our experience, and account for the correlations in terms of the structure of the Hilbert space. ${ }^{28}$ This claim would seem to be justified by the fact that entanglement is unanimously considered to be the main conceptual novelty with respect to the classical world.

If this hypothesis were correct, the question that remains open in the philosophy of quantum mechanics would not be the non-separability of the measurement outcomes - which would have to be regarded as unexplainable and primitive - but rather the emergence of a classical world. Currently, this problem is tackled via dynamical reduction models, or, in Everettian approaches, via decoherence, that allegedly explains to local observers the appearance of quasi-classical worlds that don't interfere. In Bohmian mechanics, the question is sometimes regarded as explaining the emergence of the three-dimensional macroscopic world out of an ontology that regards as fundamental the $3 \mathrm{~N}$ space in which the wave function allegedly evolves (Albert and Ney 2013). This paper is, in part, also an invitation to explore whether the explanatory switch suggested above is valid also in the quantum case.

\footnotetext{
${ }^{28}$ Dorato and Felline (2011) is a first stab in this direction.
} 
References

Achinstein P. (1983), The nature of explanation, Oxford University Press, Oxford.

Albert D. and Ney A. eds. (2013), The wave function, Oxford University Press, Oxford.

Balashov Y. Janssen M. (2003), "Presentism and Relativity", British Journal for the Philosophy of Science, 54, pp. 327-346.

Batterman R. (2010), "On the Explanatory Role of Mathematics in Empirical Science", British Journal for the Philosophy of Science, 61, pp. 1-25.

Belot G. and Earman J. (2001), "Pre-Socratic Quantum Gravity," in C. Callender and N. Huggett (eds.), Physics Meets Philosophy at the Planck Scale, Cambridge University Press, pp. 213-255.

Bigelow J., Ellis B. Pargetter R. (1988), "Forces", Philosophy of Science, 55 pp. 614-630.

Bueno O. and French S (2012), "Are there mathematical explanations of physical phenomena", British Journal for the Philosophy of Science , 63, pp. 85-113.

Brandon R. N. (2006), "The principle of drift: biology's first law”, Journal of Philosophy, 103, pp. 319-335.

Brown, H. (2005), Physical Relativity: Spacetime Structure from a Dynamical Perspective, Clarendon Press, Oxford University Press.

Brown H. and Pooley O. (2006), "Minkowski Space-Time: A glorious Non-Entity", in Dennis Dieks (ed.), The Ontology of Spacetime. Elsevier, pp. 67-91.

Clagett, M. (1961), The Science of Mechanics in the Middle Ages, Madison, University of Wisconsin Press.

Clifton, R. (1998), "Structural Explanation in Quantum Theory", http://philsciarchive.pitt.edu/archive/00000091/00/explanation-in-QT.pdf.

Colyvan, M. (1998): 'Can the Eleatic Principle Be Justified?', Canadian Journal of Philosophy, 28, pp. 313-36.

De Regt H., \& Dieks D., (2005), "A Contextual Approach to Scientific Understanding", Synthese, 144, 1, 2005, pp. 137-170.

Di Salle R. (2006), Understanding Spacetime. Cambridge University Press, Cambridge.

Dorato M. (2000), "Substantivalism, relationism and structural spacetime realism," Foundations of Physics, 30 (10): 1605-1628.

Dorato M. and Felline L. (2010), "Structural explanations in Minkowski spacetime: which account of models?" in V. Petkov (ed.) Space, Time, and Spacetime - Physical and Philosophical Implications of Minkowski's Unification of Space and Time, Springer, Berlin, Heidelberg, New York, pp.193-209.

Dorato M. Felline L. (2011), "Scientific Explanation and Scientific Structuralism", in A. Bokulich and P. Bokulich (eds.), Scientific Structuralism, Springer, pp.161-176.

Dowe P. (2000), Physical Causation, Cambridge: Cambridge University Press.

Earman J. \& M. Friedman (1973), "The Meaning and Status of Newton's Law of Inertia and the Nature of Gravitational Forces". Philosophy of Science 40 (3), pp. 329-359.

Einstein A. (1919), “Time, Space, and Gravitation.” Times (London). 28 November 1919, 1314.

Fine A. (1989), "Do Correlations need to be explained", in Cushing J. and McMullin E. (eds.) Philosophical Consequences of Quantum Theory, Notre Dame University Press, Notre Dame, pp.175-194. 
Friedman, M., (1974), 'Explanation and Scientific Understanding', Journal of Philosophy, 71: $5-19$.

Frisch M. (2009), 'Causality and Dispersion: A reply to John Norton', British Journal for Philosophy of Science, 60, p.487-495.

Friedman M. (1983), Foundations of Spacetime Theories, Princeton University Press.

Grant, E. (1977), Physical Science in the Middle Ages, Cambridge University Press, Cambridge.

Hall, N. (2004) "Two Concepts of Causation" in J. Collins, N. Hall, and L. A. Paul, eds., Causation and Counterfactuals. Cambridge, MA: The MIT Press, pp. 181-204

Hertz H. (1894), Die Prinzipien der Mechanik, Geest \& Portig, Leipzig.

Hughes, R. I. G. (1997): 'Models and Representation', Philosophy of Science, 64, S325-36.

Hughes (1989a), Bell's theorem, ideology, and structural explanation, in Cushing, J. and

McMullin, J. (eds.), Philosophical Consequences of Quantum Theory, Notre Dame, pp. 195207.

Hughes R.I.G. (1989b), The Structure and Interpretation of Quantum Mechanics, Harvard University Press.

Janiak, A. (ed.), 2004, Newton: Philosophical Writings, Cambridge: Cambridge University Press.

Janssen M. (2002), "Reconsidering a scientific revolution, the case of Einstein versus Lorentz", Physics in perspective, pp. 421-446.

Janssen M. (2002a), "COI stories: Explanation and evidence in the history of science", Physics in perspective, 10, p.457-520.

Janssen M. (2009), "Drawing the line between kinematics and dynamics in special relativity", Studies in History and Philosophy of Modern Physics, pp. 26-52.

Kitcher, P. (1989): 'Explanatory Unification and the Causal Structure of the World', in P. Kitcher and W. Salmon (eds.), Scientific Explanation, Minneapolis: University of Minnesota Press, pp. 410-505.

Kuhn T. (1970), The Structure of Scientific Revolutions. Chicago University Press, Chicago, Second Enlarged Edition.

Kuhn, T. (1977) "Concept of Cause in the Development of Physics", in The Essential Tension, University of Chicago Press, Chicago, Ch. 2., pp. 21-30.

Lange M. (2009), Laws and Lawmakers, Oxford: Oxford University Press.

Lange M. (2013a) How to Explain the Lorentz Transformations', in S. Mumford and M. Tugby (eds.), Metaphysics and Science, Oxford: Oxford University Press, pp.73-98.

Lange M. (2013b), "What makes a scientific explanation distinctively mathematical?", British Journal for the Philosophy of Science, 64, pp. 485-511.

Mach E. (1874), Die Geschichte und die Wurzel des Satzes von der Erhaltung der Arbeit, Prague. Eng. Transl. by P. Jourdain, The History And Root Of The Principle Of Conservation Of Energy, Chicago, The Open Court, 1911.

Mancosu, P. (2008): 'Mathematical Explanation: Why it Matters', in The Philosophy of Mathematical Practice, Oxford: Oxford University Press, pp. 134-50.

McMullin, E. (1978) "Structural Explanations", American Philosophical Quarterly, 15, pp. 139-48

Nerlich G. (1994), The Shape of Space, Cambridge University Press,2nd ed.

Nerlich G. (2010), "Why spacetime is not a hidden cause: a Realist story", in V. Petkov (ed.) Space, Time, and Spacetime: Physical and Philosophical Implications of Minkowski's Unification of Space and Time, Springer, Berlin, Heidelberg, New York, pp. 181-191.

Newton I. (1726 [1999]), The Principia: Mathematical Principles of Natural Philosophy, transl. I.B. Cohen, and A. Whitman. University of California Press, Berkeley and Los Angeles. 
North J. (2009), “The Structure of Physics: a case study, Journal of Philosophy 106 (2009): $57-88$.

Norton J.D. (2008), "Why constructive relativity fails", British Journal for Philosophy of science, 59 , pp. 821-83.

Pincock C. (2007), “A Role for Mathematics in the Physical Sciences”, Nôus, 41, pp. 253275.

Quine W. V. and Ullian J. S. (1978), The Web of Belief, McGraw-Hill.

Reutlinger A. (2012) "Getting Rid of Interventions", Studies in the History and Philosophy of Science Part C 43 (4): 787-795.

Russell, B. (1992) "On the Notion of Cause," orig. 1912, in J. Slater (ed.), The Collected Papers of Bertrand Russell v.6: Logical and Philosophical Papers 1909-1913. London: Routledge Press, pp. 193-210.

Schaffer, J. (2000) "Causation by Disconnection," Philosophy of Science, 67: 285-300

Schaffer, J., "The Metaphysics of Causation", The Stanford Encyclopedia of Philosophy (Winter 2013 Edition), Edward N. Zalta (ed.), URL = $<$ http://plato.stanford.edu/archives/win2013/entries/causation-metaphysics/>

Sellars W. (1962), "Philosophy and the Scientific Image of Man", in Colodny R. (ed.), Frontiers of Science and Philosophy, University of Pittsburgh Press, Pittsburgh, pp. 3578.

Salmon W. (1989), Forty Years of Scientific Explanation, University of Minnesota Press, Minneapolis.

Stein, H. (1989), "Yes but...some skeptical remarks on the realism antirealism debate", Dialectica, 43, 1-2, pp. 47-65

Toulmin S. (1961), Foresight and Understanding, Harper and Row, New York.

Wald R. (1984), General Relativity, University of Chicago Press, Chicago.

Woodward, J. (2003), Making Things Happen: A Theory of Causal Explanation, Oxford: Oxford University Press. 